\title{
Inhibitory Activity of Iron Chelators ATA and DFO on MCF-7 Breast Cancer Cells and Phosphatases PTP1B and SHP2
}

\author{
ALICJA KUBAN-JANKOWSKA ${ }^{1}$, KAMLESH K. SAHU ${ }^{2}$, MAGDALENA GORSKA-PONIKOWSKA ${ }^{1}$, \\ JACK A. TUSZYNSKI ${ }^{3}$ and MICHAL WOZNIAK ${ }^{1}$ \\ ${ }^{1}$ Department of Medical Chemistry, Medical University of Gdansk, Gdansk, Poland; \\ ${ }^{2}$ Department of Medical Microbiology and Immunology, University of Alberta, Edmonton, Canada; \\ ${ }^{3}$ Department of Oncology, University of Alberta, Edmonton, Canada
}

\begin{abstract}
Background: Rapidly-dividing cancer cells have higher requirement for iron compared to non-transformed cells, making iron chelating a potential anticancer strategy. In the present study we compared the anticancer activity of uncommon iron chelator aurintricarboxylic acid (ATA) with the known deferoxamine (DFO). Materials and Methods: We investigated the impact of ATA and DFO on the viability and proliferation of MCF-7 cancer cells. Moreover we performed enzymatic activity assays and computational analysis of the ATA and DFO effects on pro-oncogenic phosphatases PTP $1 B$ and SHP2. Results: ATA and DFO decrease the viability and proliferation of breast cancer cells, but only ATA considerably reduces the activity of PTPIB and SHP2 phosphatases. Our studies indicated that ATA strongly inactivates and binds in the $P T P 1 B$ and SHP2 active site, interacting with arginine residue essential for enzyme activity. Conclusion: We confirmed that iron chelating can be considered as a potential strategy for the adjunctive treatment of breast cancer.
\end{abstract}

Breast cancer is one of the most common female tumors worldwide and the number of breast cancer cases is continually growing (1). Because of the genetic and environmental factors, breast cancer is more often becoming a problem of younger women (2). Previous studies showed that the development of breast cancer is involved with tyrosine phosphorylation pathway disorders (3). Tyrosine phosphorylation is controlled by careful

This article is freely accessible online.

Correspondence to: Alicja Kuban-Jankowska, Medical Chemistry Department, Medical University of Gdansk, Gdansk, Poland. Debinki 1, 80-210 Gdansk, Poland. Tel: +48 583491450, Fax: +48 583491456, e-mail: alicjakuban@gumed.edu.pl

Key Words: Breast cancer, iron chelators, ATA, DFO, PTP1B, SHP2. balance of activity of tyrosine kinases and tyrosine phosphatases (4). Protein tyrosine phosphatases PTP1B and SHP2 are overexpressed in breast cancer cell lines and promote tumor growth $(5,6)$. Phosphatases PTP1B and SHP2 may constitute promising targets for the development of new anticancer diagnostic and therapeutic strategies (7). Previous studies have shown that aurintricarboxylic acid (ATA) (Figure 1A) is a very effective inhibitor of protein tyrosine phosphatases YopH and CD45 $(8,9)$. ATA is not only known to inhibit protein tyrosine phosphatases, but also nucleic acid binding enzymes, such as reverse transcriptase, DNA and RNA polymerase, topoisomerase and nuclease (10). Taking the above studies into consideration, it is readily concluded that ATA is a remarkable compound, which should be seriously considered as a drug for the treatment of numerous diseases, with a particular focus on cancer. The previous studies confirmed that aurintricarboxylic acid decreases proliferative potential of human ovarian SKOV3 as well as human breast cancer MCF-7 and T47D cells $(11,12)$. Recently it has been also found that treatment with ATA enhanced glioma cell sensitivity to both the chemotherapeutic agent temozolomide and radiation-induced cell death (13).

ATA is a polyaromatic carboxylic acid derivative exhibiting polyanionic and metal chelating properties, which may be essential for anti-cancer activity (14), as iron chelators are considered as potential anti-tumor agents (15). Recent studies showed that iron deprivation is promising strategy for cancer therapy investigation (16). Iron is an essential element for cell physiology being critical for DNA synthesis. Rapidly-dividing cancer cells have higher requirement for iron comparing to their non-transformed counterparts, making them particularly sensitive to iron depletion. Deferoxamine (DFO) (Figure 1B) was the most studied iron chelator, and the first one introduced into clinical practice. DFO is used for the treatment of iron-overload disease and to remove excess iron that accumulates with chronic blood transfusions (17), but it has also been shown to possess anticancer activity (18). 
A
Aurintricarboxylic acid (ATA)<smiles>CC1=CC(=C(c2ccc(O)c(C(=O)O)c2)c2ccc(O)c(C(=O)O)c2)C=C(C(=O)O)C1=O</smiles>

B

\section{Deferoxamine}<smiles>CC(=O)N(O)CCCCCNC(=O)CCC(=O)N(O)CCCCCNC(=O)CCC(=O)N(O)CCCCCN</smiles>

Figure 1. Structures of $(A)$ aurintricarboxylic acid and $(B)$ deferoxamine.

Here we present the studies on the effect of iron chelators ATA and DFO on the viability and proliferation of MCF-7 breast cancer cells. We also compare the impact of ATA and DFO on the enzymatic activity of pro-oncogenic PTP1B and SHP2 phosphatases.

\section{Materials and Methods}

Reagents. Phosphatases PTP1B (No. SRP0215) and SHP2 (No. SRP0217) were obtained from Sigma Aldrich. MCF-7 cell line was purchased from The European Collection of Cell Cultures (ECACC). Aurintricarboxylic acid (No. A1895), deferoxamine (No. D9533), cell media, supplements and other reagents were obtained from Sigma Aldrich. Cell proliferation assay kit was obtained from BioAssay Systems.

Cell culture. The cells were cultured in DMEM medium supplemented with $10 \%$ fetal bovine serum, $100 \mu \mathrm{g} / \mathrm{ml}$ penicillin/streptomycin and $2 \mathrm{mM}$ L-glutamine. The culture was maintained at $37^{\circ} \mathrm{C}$ and in an atmosphere containing $5 \% \mathrm{CO}_{2}$. The cell culture density was kept to maximum $1 \times 10^{6}$ cells $/ \mathrm{ml}$. At least every two days the medium was replaced with the fresh one, and the cells were counted and reseeded to maintain the recommended density.

Cell viability test. The cells $\left(1 \times 10^{6}\right.$ cells $\left./ \mathrm{ml}\right)$ untreated (control) or treated with solutions of ATA and DFO after the appropriate incubation time were suspended in solution of $5 \mathrm{mg} / \mathrm{mL}$ MTT(3-[4,5dimethylthiazol-2-yl]-2,5-diphenyltetrazolium bromide) in DMEM without phenol red. The $100-\mu \mathrm{l}$ samples were incubated for 3 to $4 \mathrm{~h}$ at $37^{\circ} \mathrm{C}$ in 96 -well plates. When the purple precipitate was clearly visible under the microscope, $100 \mu \mathrm{l}$ of DMSO was added to each well and the plate was left in the dark for $15 \mathrm{~min}$. The absorbance at $590 \mathrm{~nm}$ was determined using a microplate reader.
Fluorescent assay for cell proliferation. The cells $\left(1 \times 10^{6}\right.$ cells $\left./ \mathrm{ml}\right)$ untreated (control) or treated with solutions of ATA and DFO after the appropriate incubation time were suspended in solution of CellQuantiBlue reagent of resazurin. The 200- $\mu$ l samples were incubated for 1 to 5 hat $37^{\circ} \mathrm{C}$ in 96 -well plates. The fluorescence intensity of highly fluorescent product (resorufin) was measured using a fluorescence reader (excitation wavelength $=530-570 \mathrm{~nm}$, emission wavelength=590-620 $\mathrm{nm}$ ).

$P T P 1 B$ and SHP2 activity assay. The solutions of the recombinant PTP1B and SHP2 phosphatase were prepared in $10 \mathrm{mM}$ HEPES buffer $\mathrm{pH}$ 7.4. The final concentration of phosphatases in reaction samples was $1.5 \mu \mathrm{g} / \mathrm{ml}(3.3 \mathrm{nM})$. The enzymes were untreated (control) or treated with solutions of oxidized and reduced lipoic acids. The assay was performed in 96-well microplates, and the final volume of each sample was $200 \mu \mathrm{l}$. The enzymatic activity of phosphatases was measured at $37^{\circ} \mathrm{C}$ at $405 \mathrm{~nm}$ on a microplate reader Jupiter (Biogenet) using DigiRead Communication Software (Asys Hitech $\mathrm{GmbH}$ ), using 2-mM chromogenic substrate paranitrophenyl phosphate ( $p$ NPP).

Docking studies. The initial structure of PTP1B was imported from the RCSB protein data bank (www.pdb.org) with code 5K9V.pdb and SHP2 with code 5EHR.pdb. The structures were minimized using Amber10:EHT force field of the Molecular Operating Environment software (MOE, Chemical Computing Group). The ligands were removed from pdb files and ATA or DFO were docked into the structures. A docking simulation was performed, where the binding site was assumed to be the active site. The side chains were kept free to move during force field refinement. Alpha PMI is the placement method used with default settings (sample per conformation $=10$, maximum poses=250). London $d G$ rescoring was used with Alpha PMI placement. Termination criteria for force field refinement were set as gradient $=0.001$ and iterations $=500$. 
A

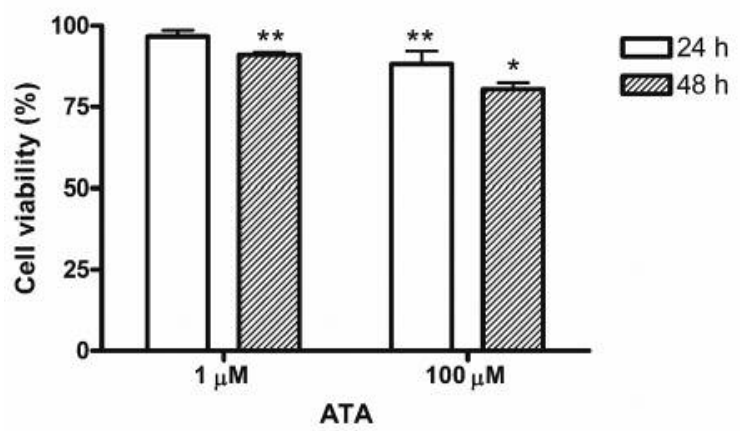

B

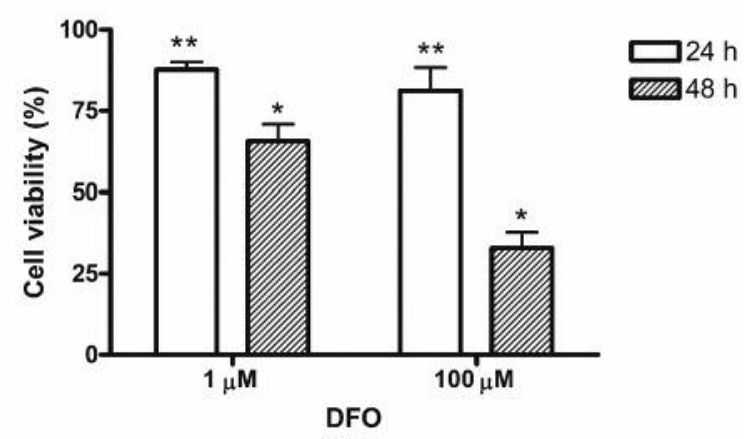

Figure 2. Cell viability of MCF-7 breast cancer cell line after treatment for 24 and $48 \mathrm{~h}$ with (A) $1 \mu M, 100 \mu \mathrm{M}$ aurintricarboxylic acid (ATA) and (B) deferoxamine (DFO) measured with MTT-based cell viability test. Data presented as percent of the control viability $(100 \%$, cells not treated), mean $\pm S D(n=3)$. One-way Anova test combined with Tukey test. ${ }^{*}$ Means were significantly different from control $(p<0.001)$. **Means were significantly different from control $(p<0.05)$.
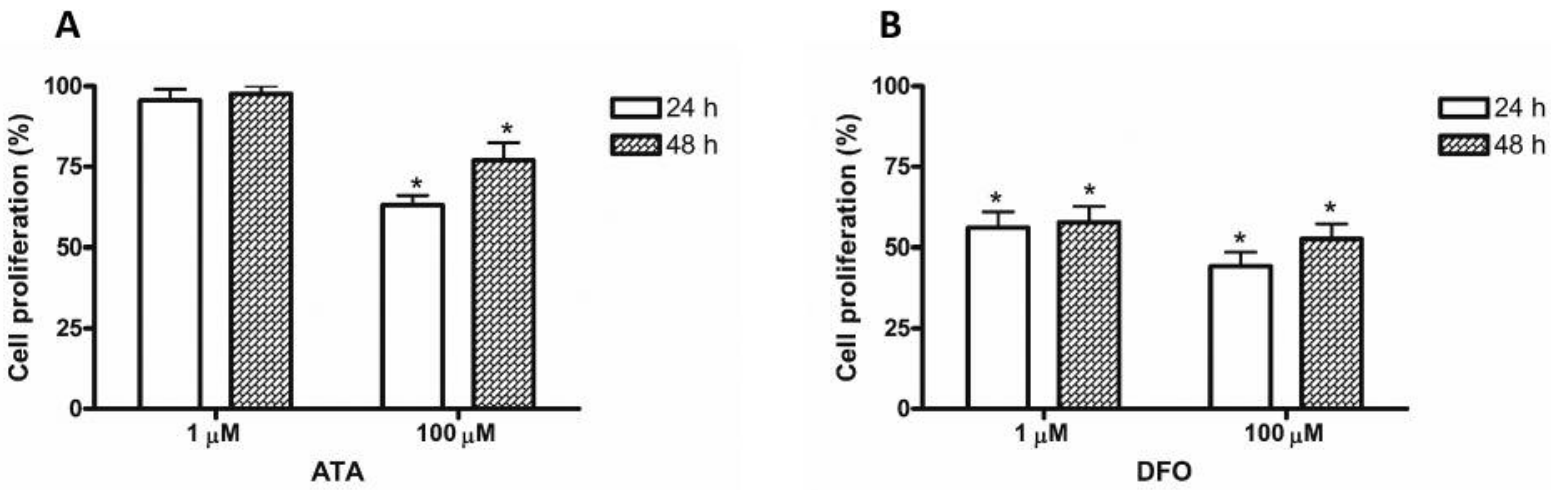

Figure 3. Cell proliferation of MCF-7 breast cancer cell line after treatment for 24 and $48 \mathrm{~h}$ with (A) $1 \mu M, 100 \mu M$ aurintricarboxylic acid (ATA) and $(B)$ deferoxamine $(D F O)$ measured with resazurin-based fluorescent cell proliferation test. Data presented as a percent of the control (100\%, cells not treated), mean $\pm S D(n=3)$. One-way Anova test combined with Tukey test. ${ }^{*}$ Means were significantly different from control ( $\left.p<0.001\right)$.

Statistical analysis. The experiments were performed at least three times. The data were applied and analyzed with GraphPad Prism (GraphPad Software v.4). Statistical analyses were performed using ANOVA combined with Tukey's test or $t$-test combined with Wilcoxon test. The data were expressed as means \pm SD. Differences between means were considered significant for $p<0.05$.

\section{Results}

ATA and DFO effect on MCF-7 breast cancer cell viability and proliferation. We evaluated the effect of ATA and DFO on the viability of MCF-7 breast cancer cell line. We found that ATA and DFO are able to decrease the cell viability in concentration-dependent manner. After $24 \mathrm{~h}$ of treatment, the viability of cells was significantly $(p<0.05)$ decreased by 1 ,
$100 \mu \mathrm{M}$ DFO and $100 \mu \mathrm{M}$ ATA (Figure 2). 1- $\mu \mathrm{M}$ ATA has no impact on cell viability, whereas $1 \mu \mathrm{M}$ DFO has already inhibitory effect $(p<0.05)$. After $48 \mathrm{~h}$ of treatment, the viability of cells was significantly $(p<0.001)$ decreased by 1 , $100 \mu \mathrm{M}$ DFO and $100 \mu \mathrm{M}$ ATA, as well as by $1 \mu \mathrm{M}$ ATA $(p<0.001)$ (Figure 2).

We measured the effect of ATA and DFO on cell proliferation with fluorescence assay and found that DFO has a stronger inhibitory effect on MCF-7 cell proliferation than ATA (Figure 3). After $24 \mathrm{~h}$ incubation time $1 \mu \mathrm{M}$ as well as $100 \mu \mathrm{M}$ DFO induced significant $(p<0.001)$ inhibition of MCF-7 cell proliferation, whereas ATA was effective only in $100 \mu \mathrm{M}$. The same effect was observed after $48 \mathrm{~h}$ incubation time, both concentrations of DFO and only $100 \mu \mathrm{M}$ ATA 

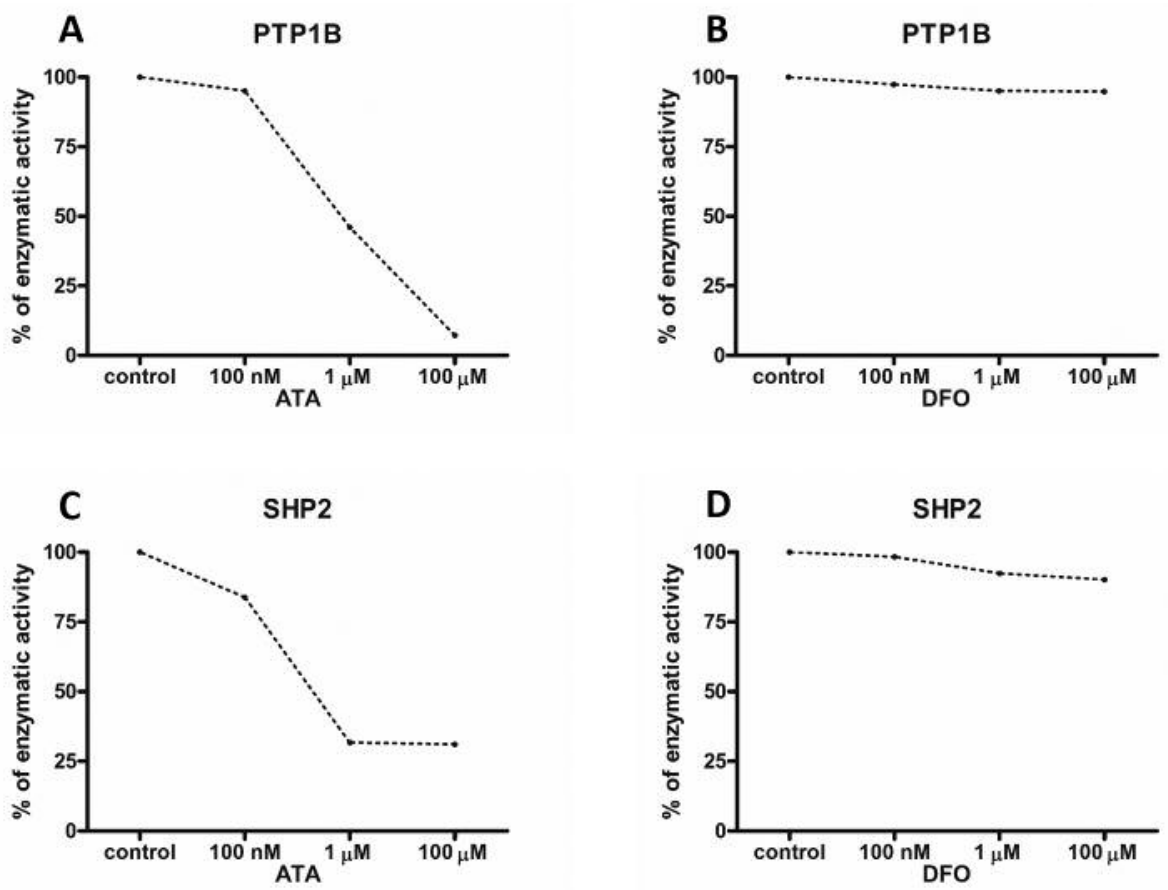

Figure 4. (A) Enzymatic activity of PTP1B phosphatase after 30 min of treatment with different concentrations of aurintricarboxylic acid (ATA). (B) Enzymatic activity of PTP1B phosphatase after 30 min of treatment with different concentrations of deferoxamine (DFO). (C) Enzymatic activity of SHP2 phosphatase after 30 min of treatment with different concentrations of aurintricarboxylic acid (ATA). (D) Enzymatic activity of SHP2 phosphatase after $30 \mathrm{~min}$ of treatment with different concentrations of deferoxamine (DFO). Data presented as percent of the control enzymatic activity $(100 \%$, cells not treated), mean $\pm S D(n=3)$.

significantly $(p<0.001)$ decreased the cell proliferation of MCF-7 cells (Figure 3).

Inhibitory effect of ATA and DFO on enzymatic activity of $P T P 1 B$, SHP2 phosphatases. In the next step, we examined the effect of ATA and DFO on enzymatic activity of protein tyrosine phosphatases PTP1B and SHP2. In order to assess the inhibitory effect of ATA and DFO on PTP1B and SHP2 phosphatases we performed an enzymatic activity assay of recombinant PTPs after treatment and 30 min incubation with tested compounds. We found that ATA and DFO are able to decrease the enzymatic activity of PTP1B and SHP2, but ATA showed much greater inhibitory activity than DFO (Figure 4). The inhibition of phosphatases was concentration-dependent. The effect of ATA was increasing relative to concentration and was slight for $100 \mathrm{nM}$ ATA, while $1 \mu \mathrm{M}$ ATA induced strong inhibition of PTP1B and SHP2 phosphatases (Figure $4 \mathrm{~A}$ and C). $100 \mu \mathrm{M}$ DFO decreased no more than $20 \%$ of the activity of PTP1B and SHP2 (Figure 4B and D).

Calculations of $I C_{50}$ values of ATA and DFO on PTPIB and SHP2 phosphatases. To compare the influence of ATA and DFO on PTP1B and SHP2 we calculate $\mathrm{IC}_{50}$ values (Table I). We used different concentrations of ATA an DFO and 30-min
Table I. The inhibitory properties of aurintricarboxylic acid (ATA) and deferoxamine (DFO) to PTP1B and SHP2 phosphatases presented as $I C_{50}$ values. $I C_{50}$ values were determined from a plot presenting inhibitor concentration versus percentage of the enzymatic activity measured as absorbance with pNPP substrate after 30 minutes incubation with inhibitors, at substrate concentration equal to Km value. Data presented as an estimated concentration from the plot.

\begin{tabular}{|c|c|c|}
\hline \multirow[b]{2}{*}{ Phosphatase } & \multicolumn{2}{|c|}{ Calculated $\mathrm{IC}_{50}$ values } \\
\hline & ATA & DFO \\
\hline PTP1B & $0.93 \pm 0.028 \mu \mathrm{M}$ & $2.54 \pm 0.68 \mathrm{mM}$ \\
\hline SHP2 & $0.517 \pm 0.021 \mu \mathrm{M}$ & $2.91 \pm 0.76 \mathrm{mM}$ \\
\hline
\end{tabular}

incubation time for phosphatases with compounds. We calculated $\mathrm{IC}_{50}$ values based on a plot presenting ATA and DFO concentration versus percentage of the enzymatic activity of recombinant PTP1B and SHP2 measured as absorbance with $p$ NPP substrate. The $p$ NPP concentration for $\mathrm{IC}_{50}$ calculations was equal to $\mathrm{K}_{\mathrm{m}}$ value determined for PTP1B and SHP2, where $K_{m}$ value is defined as substrate concentration at which enzyme activity is half-maximal. 

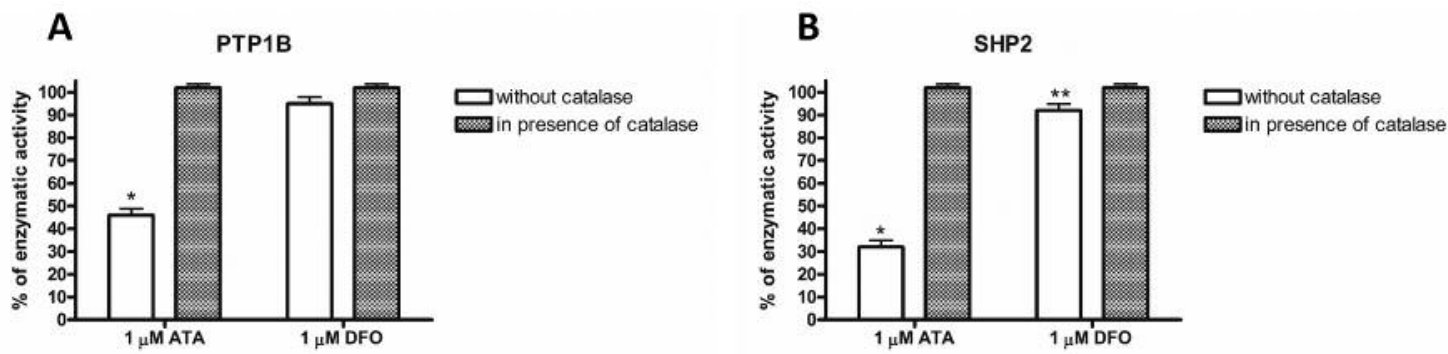

Figure 5. (A) Enzymatic activity of PTP1B phosphatase after 30 min of treatment with $1 \mu M$ aurintricarboxylic acid (ATA) and deferoxamine (DFO) in presence and absence of catalase. (B) Enzymatic activity of SHP2 phosphatase after 30 min of treatment with $1 \mu M$ aurintricarboxylic acid (ATA) and deferoxamine (DFO) in presence and absence of catalase. Data are presented as percent of the control enzymatic activity (100\%, cells not treated), mean $\pm S D(n=3)$. One-way Anova test combined with Tukey test. $*$ Means were significantly different from control $(p<0.001)$. $* * M e a n s$ were significantly different from control $(p<0.05)$.

The obtained results indicated that DFO is able to decrease the enzymatic activity of selected phosphatases, but is much more weaker than ATA. DFO was effective in a concentration in a range of about 2-3 $\mathrm{mM}$ (Table $\mathrm{I}$ ). The calculated $\mathrm{IC}_{50}$ value of ATA showed that it is strong inhibitor of PTP1B and SHP2 phosphatases and is effective in concentration below $1 \mu \mathrm{M}$. We observed the tendency that ATA induced more selective inhibition of SHP2 than PTP1B phosphatase (Table I).

ATA losses inhibitory activity in the presence of catalase. In the next step we performed an enzymatic activity assay of recombinant $\mathrm{PTP} 1 \mathrm{~B}$ and SHP2 in presence and absence of catalase to evaluate if the hydrogen peroxide generation is involved in the inactivation. As we expected from our previous studies (8), the inhibitory effect of ATA was abolished in a presence of catalase (Figure 5). We found that catalase has no impact on inhibition caused by DFO (Figure 5).

Docking studies of ATA and DFO in PTP1B and SHP2 binding site. ATA and DFO molecules were docked into the 3D structures of PTP1B and SHP2 in order to investigate the possibility of binding and conformation. We performed flexible docking and retained top 30 conformations from docking runs. In all 30 conformations ATA and DFO are bound to the active site of PTP1B and SHP2, as shown in Figure 6. The docking studies showed that ATA and DFO are not precluded to bind in the active site of PTP1B and SHP2. Both compounds can be easily accommodated inside the binding site and binds specifically in a catalytic center of PTP1B and SHP2.

The binding simulations of PTP1B and SHP2 with ATA and $D F O$. To study the binding conformation of ATA and DFO in the PTP1B and SHP2 active sites we identified top scoring poses from docking studies. The interactions of ATA and DFO in the PTP1B and SHP2 binding site are presented as a PLIF diagrams (protein ligand interaction fingerprints) in
Figure 7. The dotted line around the molecule shows solvent contact and dotted arrows represent hydrogen bonds between amino acid residues from PTPs and inhibitor. As shown in the PLIF diagrams (Figure 7A and C), ATA is able to utilize its carboxyl groups to interact with active site arginine residues of PTP1B (Arg221) and SHP2 (Arg465), essential for their enzymatic activity. We have not observed any interactions of DFO with essential residues in PTP1B and SHP2 active site (Figure 7B and D).

\section{Discussion}

Breast cancer is found to be one of the most common cancers among women worldwide and usually undergoes with complicated etiology and multiple organ metastasis. Its therapy includes surgery, radiotherapy or adjuvant chemotherapy. There are many studies showing the involvement of tyrosine phosphorylation pathways disorders in many types of cancer, among others, tyrosine phosphatases PTP1B and SHP2 in induction of breast cancer (3). PTP1B dephosphorylates tyrosine kinases, i.e. HER1/EGFR, Src, JAK and STAT, involved in the development of breast cancer $(5,20)$. Mutations of protein tyrosine phosphatase SHP2 have been found in breast cancer cells, and thus, SHP2 can be a promising target in anticancer therapy (6). Due to the key part of phosphatases PTP1B and SHP2, inhibition of their activity may constitute a promising strategy for the treatment of breast cancer (7).

Previous studies showed that ATA can be considered an inhibitor of pro-oncogenic PTP1B and SHP2, as it is very effective inhibitor of other protein tyrosine phosphatases $(8$, 9). Here we showed that ATA can significantly decrease the activity of PTP1B as well as SHP2 (Figure 4A and C), and is much more effective that DFO (Figure 4B and 4D). The differences in inhibitory effect can be due to different binding conformation and interactions of ATA and DFO in the active site of PTP1B and SHP2. The docking analysis we performed 

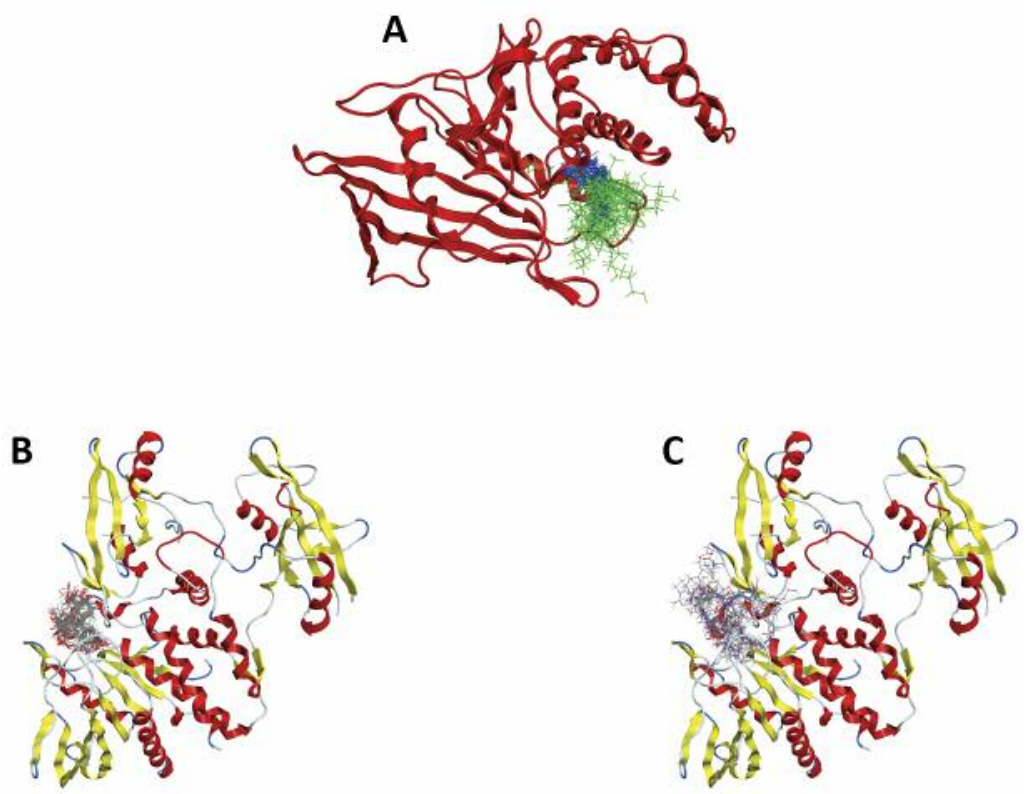

Figure 6. Computational analysis of aurintricarboxylic acid (ATA) and deferoxamine (DFO) binding with PTP1B and SHP2. (A) The site of binding for top 30 conformations of ATA (blue) and DFO (green) obtained from docking of ATA and DFO into the PTP1B structure. In each conformation ATA and DFO bind in the PTP1B active site. (B) The site of binding for top 30 conformations of ATA obtained from docking of ATA into the SHP2 structure. In each conformation ATA binds in the SHP2 active site. (C) The site of binding for top 30 conformations of DFO obtained from docking of DFO into the SHP2 structure. In each conformation DFO binds in the SHP2 active site.

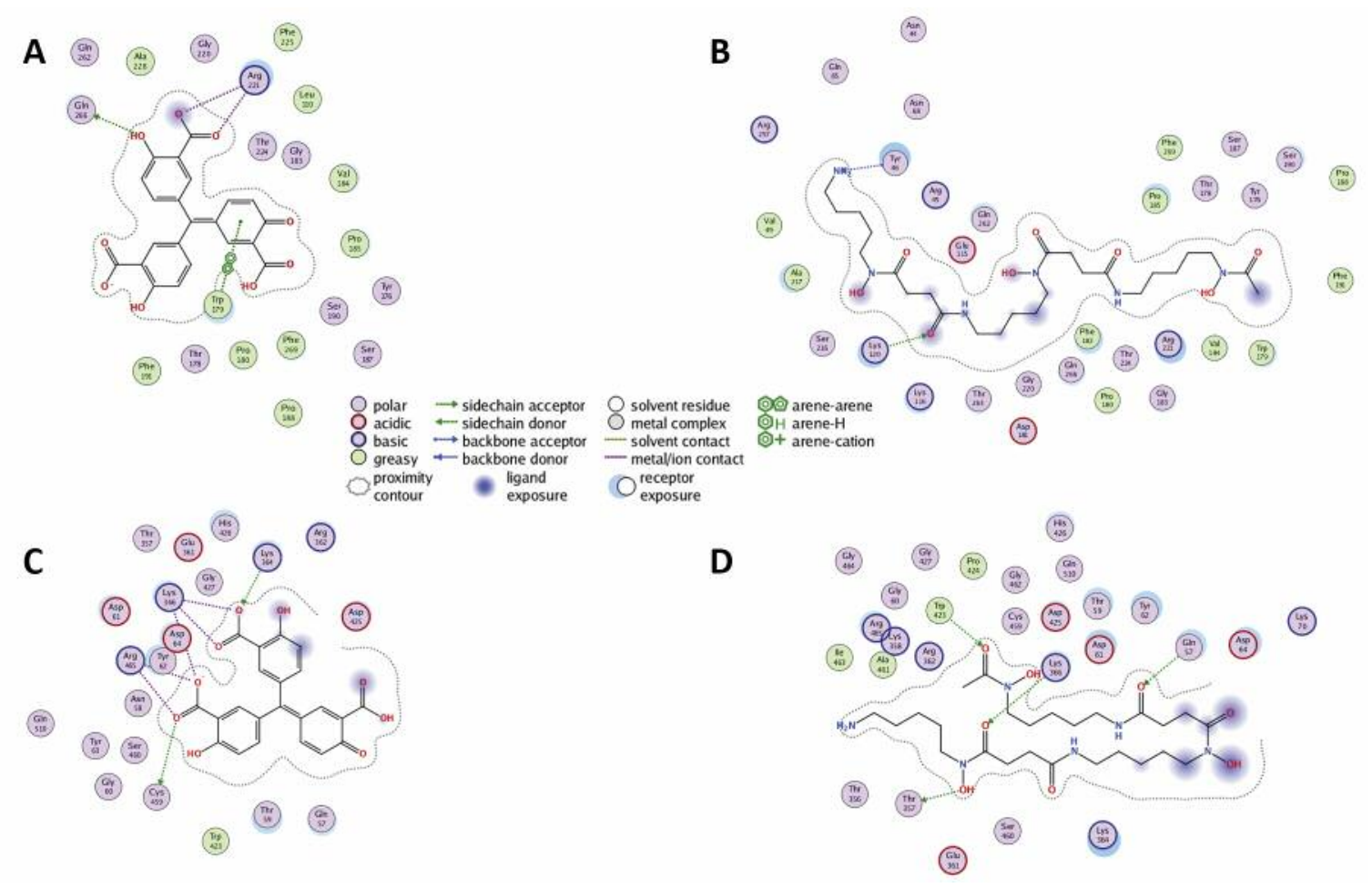

Figure 7. (A) The PLIF diagram for the best binding pose of aurintricarboxylic acid (ATA) in the PTP1B active site. In predicted binding pose, the carboxyl group of ATA interacts with active-site arginine residues of PTP1B (Arg221). (B) The PLIF diagram for the best binding pose of deferoxamine (DFO) in the PTP1B active site. C) The PLIF diagram for the best binding pose of ATA in the SHP2 active site. In predicted binding pose, the carboxyl group of ATA interacts with active site arginine residues of SHP2 (Arg465). (D) The PLIF diagram for the best binding pose of DFO in the SHP2 active site. 
allowed us to observe, that although both compounds are not precluded to bind the active sites of PTP1B and SHP2, only ATA interacts with the catalytic arginine residue (Figure 7A and $\mathrm{C}$ ). The positively-charged arginine residue of the PTP1B and SHP2 active site is likely to attract the negatively charged carboxyl groups from ATA. The arginine residue (Arg221 in PTP1B and Arg465 in SHP2) in the catalytic pocket of tyrosine phosphatases is responsible for ligand binding and is essential for enzyme activity (21-23). Moreover, the inhibitory effect of ATA was abolished in a presence of catalase (Figure 5A and B), which is allowing us to conclude that inactivation of PTP1B and SHP2 can be due to activesite directed oxidation of catalytic cysteine residue, as previously observed for ATA-induced inactivation of bacterial YopH phosphatase (9).

ATA was already found to have an inhibitory effect on cancer cells and enhance sensitivity for chemotherapy (13). ATA is known to possess metal-chelating properties, which could be important mechanism of action of ATA in anti-cancer treatment (14). Previous studies showed that compounds with iron chelating properties may have significant impact in cancer metabolism and can be considered in chemotherapy $(16,19)$. Recently we have shown, among other groups, that lipoic acid (LA) has inhibitory effect on MCF-7 cell viability and proliferation (24-27), as well as on activity of pro-oncogenic phosphatases PTP1B and SHP2 (27). Lipoic acid is also known to has metal-chelating properties, which can be possible anticancer mechanism of LA action (28).

Rapidly-dividing cancer cells have a higher iron requirement than cells dividing properly. Tumor cells are thus sensitive to decrease of iron (15). This enables the use of iron chelators as a new concept for the design of cancer treatments. The study of iron chelators as anti-tumor agents is still in its infancy. Iron is important for cellular proliferation and this is demonstrated by observations that iron-depletion results in cell cycle arrest and also apoptosis (29). In addition, many iron chelators are known to inhibit ribonucleotide reductase, the iron-containing enzyme that is the rate-limiting step for DNA synthesis (30).

In this study, we found inhibitory effect of uncommon iron chelator ATA and well-known DFO on cancer cell viability and proliferation using breast cancer cell line MCF-7 model. The anticancer mechanism of ATA needs to be further evaluated. However, ATA was determined to inhibit TWEAKinduced glioma cell chemotactic migration and invasion via inhibition of Rac1 activation (13). While, DFO was found to have growth-arresting and apoptosis-inducing effect via modification of the VCAM-1 adhesion molecule expression (31). ATA and DFO are membrane-permeable ferric chelators. Their moderate anti-proliferative effect on the breast cancer cell line MCF-7 may be interpreted as a consequence of deprivation of ferric ion. Some of the anti-cancer compounds utilized in breast cancer chemotherapy, e.g. dasatinib, saracatinib or bosutinib, are inhibitors of Src kinases, which in turn are dephosphorylated by PTP1B phosphatase (32). Increased levels and activity of Src kinases are widely reported in human cancers including breast cancer, and its important role in oncogenic processes makes it a potential target for therapeutic intervention (33). Inhibition of the activity of PTP1B by ATA alters the regulatory pathway and can lead to inactivation of PTP1B-targeted proteins, i.a. Src kinases. Previous studies showed that anti-tumor effect of $\mathrm{Fe}$ chelators, such as DFO, may be partly due to $\mathrm{N}$-myc downstream regulated gene-1 (Ndrg-1) up-regulation, which reduces cancer cell growth and metastasis in breast, colon, prostate and pancreatic cancer (34). Further studies are needed to investigate if the inhibitory effect of ATA and DFO on viability and proliferation of MCF-7 cells involves upregulation of Ndrg-1 gene.

In summary, we showed that ATA and DFO decrease the viability of breast cancer cells and have inhibitory effect on enzymatic activity of potentially oncogenic phosphatases PTP1B and SHP2. Our studies confirmed that ATA and DFO can be considered potential agents for the treatment of breast cancer, possibly as an adjuvant therapy with other known systemic therapeutic agents. Moreover, many anti-cancer drugs induce generation of reactive oxygen species (ROS) (35). ATA and DFO have been reported to decrease the intracellular ROS level and probably inhibition of Fenton reaction (36). Thus, ATA and DFO apart from their inhibitory effect on tumor cells, could scavenge free radicals and ROS produced by systemic anticancer agents.

\section{Conflicts of Interest}

The Authors declare that they have no conflict of interest. This article does not contain any studies with human participants or animals performed by any of the authors.

\section{Funding}

We acknowledge financial support from the project IP2015 038774 from Polish Ministry of Science and Higher Education.

\section{References}

1 Da Costa GG, Gomig TH, Kaviski R, Santos Sousa K, Kukolj C, De Lima RS, De Andrade Urban C, Cavalli IJ and Ribeiro EM: Comparative proteomics of tumor and paired normal breast tissue highlights potential biomarkers in breast cancer. Cancer Genomics Proteomics 12: 251-261, 2015.

2 Wei XQ, Li X, Xin XJ and Tong ZS: Clinical features and survival analysis of very young $($ age $<35)$ breast cancer patients. Asian Pac J Cancer Prev 14: 5949-5952, 2013.

3 Nunes-Xavier CE, Martin-Perez J, Elson A and Pulido R: Protein tyrosine phosphatases as novel targets in breast cancer therapy. Biochim Biophys Acta 1836: 211-226, 2013.

4 Tonks NK: Protein tyrosine phosphatases: from genes, to function, to disease. Nat Rev Mol Cell Biol 7: 833-846, 2006. 
5 Balavenkatraman KK, Aceto N, Britschgi A, Mueller U, Bence KK, Neel BG and BentiresAlj M: Epithelial protein-tyrosine phosphatase $1 \mathrm{~B}$ contributes to the induction of mammary tumors by HER2/Neu but is not essential for tumor maintenance. Mol Cancer Res 9: 1377-1384, 2011.

6 Aceto $\mathrm{N}$ and Bentires-Alj M: Targeting protein-tyrosine phosphatases in breast cancer. Oncotarget 3: 514-515, 2012.

7 Scott LM, Lawrence HR, Sebti SM, Lawrence NJ and Wu J: Targeting protein tyrosine phosphatases for anticancer drug discovery. Curr Pharm Des 16: 1843-1862, 2010.

8 Liang F, Huang Z, Lee SY, Liang J, Ivanov MI, Alonso A, Bliska JB, Lawrence DS, Mustelin T and Zhang ZY: Aurintricarboxylic acid blocks in vitro and in vivo activity of YopH, an essential virulent factor of Yersinia pestis, the agent of plague. J Biol Chem 278: 41734-41741, 2003.

9 Kuban-Jankowska A, Sahu KK, Niedzialkowski P, Gorska M, Tuszynski JA, Ossowski T and Wozniak M: Redox process is crucial for inhibitory properties of aurintricarboxylic acid against activity of YopH - virulence factor of Yersinia pestis. Oncotarget 6: 18364-18373, 2015.

10 Bardhan M, Chowdhurry J and Ganguly T: Investigations on the interactions of aurintricarboxylic acid with bovine serum albumin: Steady state/time resolved spectroscopic and docking studies. J Photochem Photobiol B 102: 11-19, 2011.

11 Fraifeld V, Seidman R, Sagi O, Muradian K and Wolfson M: Aurintricarboxylic acid decreases proliferative potential of SKOV3 and MCF-7 human carcinoma cells. Anticancer Res 21: 1975-1978, 2001.

12 Dinda S, Kodali-Gali S, Sevilla L, Burkley M, Hurd C and Moudgil VK: Inhibition of proliferation of T47D human breast cancer cells: alterations in progesterone receptor and p53 tumor suppressor protein. Mol Cell Biochem 175: 81-89, 1997.

13 Ross A, Dhruv HD, Mathews IT, Inge LJ, Tuncali S, Hartman LK, Chow D, Millard N, Yin HH, Kloss J, Loftus JC, Winkles JA, Berens ME and Tran NL: Identification of aurintricarboxylic acid as a selective inhibitor of the TWEAK-Fn14 signaling pathway in glioblastoma cells. Oncotarget 8: 12234-12246, 2017.

14 Sharma RK, Chopra S, Sharma SD, Pande V, Ramos MJ, Meguro $\mathrm{K}$, Inoue $\mathrm{J}$ and Otsuka M: Biological evaluation, chelation, and molecular modeling studies of novel metal-chelating inhibitors of NF-KB-DNA binding: structure activity relationships. J Med Chem 49: 3595-3601, 2006.

15 Richardson DR, Kalinowski DS, Lau S, Jansson PJ and Lovejoy DB: Cancer cell iron metabolism and the development of potent iron chelators as anti-tumor agents. Biochim Biophys Acta 1790: 702-717, 2009.

16 Yang C, Ma X, Wang Z, Zeng X, Hu Z, Ye Z and Shen G: Curcumin induces apoptosis and protective autophagy in castration-resistant prostatę cancer cells through iron chelation. Drug Des Dev Ther 11: 431-439, 2017.

17 Song TS, Hsieh YW, Peng CT, Chen TL, Lee HZ, Chung JG and Hour MJ: Combined versus monotherapy or concurrent therapy for treatment of thalassaemia. In Vivo 28: 645-649, 2014.

18 Hoke EM, Maylock CA and Shacter E: Desferal inhibits breast tumor growth and does not interfere with the tumoricidal activity of doxorubicin. Free Radic Biol Med 39: 403-411, 2005.

19 Shen L, Zhao HY, Du J and Wang F: Anti-tumor activities of four chelating agents against human neuroblastoma cells. In Vivo 19: 233-6, 2005.
20 Zhu S, Bjorge JD and Fujita DJ: PTP1B contributes to the oncogenic properties of colon cancer cells through Src activation. Cancer Res 67: 10129-10136, 2007.

21 Guo XL, Shen K, Wang F, Lawrence DS and Zhang ZY: Probing the molecular basis for potent and selective protein-tyrosine phosphatase 1B inhibition. J Biol Chem 277: 41014-41022, 2002.

22 Chen L, Sung SS, Yip ML, Lawrence HR, Ren Y, Guida WC, Sebti SM, Lawrence NJ and Wu J: Discovery of a novel shp2 protein tyrosine phosphatase inhibitor. Mol Pharmacol 70: 562-570, 2006.

23 Hellmuth K, Grosskopf S, Lum CT, Würtele M, Röder N, von Kries JP, Rosario M, Rademann J and Birchmeier W: Specific inhibitors of the protein tyrosine phosphatase Shp2 identified by highthroughput docking. Proc Natl Acad Sci USA 105: 7275-7280, 2008.

24 Bingham PM, Stuart SD and Zachar Z: Lipoic acid and lipoic acid analogs in cancer metabolism and chemotherapy. Expert Rev Clin Pharmacol 7: 837-846, 2014.

$25 \mathrm{Li}$ BJ, Hao XY, Ren GH and Gong Y: Effect of lipoic acid combined with paclitaxel on breast cancer cells. Genet Mol Res 14: 17934-17940, 2015.

26 Kono Y, Inomata M, Hagiwara S, Hiratsuka T, Suzuki K, Koga H, Shiraishi N, Noguchi T and Kitano S: Antiproliferative effects of a new $\alpha$-lipoic acid derivative, DHL-HisZnNa, in HT29 human colon cancer cells in vitro. Expert Opin Ther Targets 16: 103-109, 2012.

27 Kuban-Jankowska A, Gorska-Ponikowska M and Wozniak M: Lipoic acid decreases the viability of breast cancer cells and activity of PTP1B and SHP2. Anticancer Res 37: 2893-2898, 2017.

$28 \mathrm{Ou}$ P, Tritschler HJ and Wolff SP: Thioctic (lipoic) acid: a therapeutic metal-chelating antioxidant? Biochem Pharmacol 50: 123-126, 1995.

29 Jiang XP, Wang F, Yang DC, Elliott RL and Head JF: Induction of apoptosis by iron depletion in the human breast cancer MCF7 cell line and the $13762 \mathrm{NF}$ rat mammary adenocarcinoma in vivo. Anticancer Res 22: 2685-2692, 2002.

$30 \mathrm{Yu}$ Y, Gutierrez E, Kovacevic Z, Saletta F, Obeidy P, Suryo Rahmanto $Y$ and Richardson DR: Iron chelators for the treatment of cancer. Curr Med Chem 19: 2689-2702, 2012.

31 Wang $\mathrm{G}$, Shen $\mathrm{G}$ and Yin T: In vitro assessment of deferoxamine on mesenchymal stromal cells from tumor and bone marrow. Environ Toxicol Pharmacol 49: 58-64, 2017.

32 Lumachi F, Chiara GB, Foltran L and Basso SM: Proteomics as a guide for personalized adjuvant chemotherapy in patients with early breast cancer. Cancer Genomics Proteomics 12: 385-90, 2015.

33 Hiscox S and Nicholson RI: Src inhibitors in breast cancer therapy. Expert Opin Ther Targets 12: 757-767, 2008.

34 Kovacevic Z, Fu D and Richardson DR: The iron-regulated metastasis suppressor, Ndrg-1: Identification of novel molecular targets. Biochim Biophys Acta 1783: 1981-1992, 2008.

35 Carlisi D, Buttitta G, Di Fiore R, Scerri C, Drago-Ferrante R, Vento $\mathrm{R}$ and Tesoriere G: Parthenolide and DMAPT exert cytotoxic effects on breast cancer stem-like cells by inducing oxidative stress, mitochondrial dysfunction and necrosis. Cell Death Dis 14: e2194, 2016.

36 Zhanyou Y, Mutsumi T and Toshiomi Y: Combined addition of glutathione and iron chelators for decrease of intracellular level of reactive oxygen species and death of Chinese hamster ovary cells. J Biosci Bioeng 95: 124-127, 2003.

Received June 13, 2017

Revised July 5, 2017

Accepted July 6, 2017 